\title{
SILVERING AND EVACUATING PYREX DEWAR FLASKS
}

\author{
By R. B. Scott, J. W. Cook, and F. G. Brickwedde
}

ABSTRACT

A method of silvering and evacuating Pyrex Dewar flasks is described. The insulating properties of silvered flasks prepared in the way described in this paper are such that even in the wide-mouth cylindrical flasks filled with liquid air near to the lip there is no boiling, the change of the liquid air to the gaseous phase taking place entirely by surface evaporation. Details of the preparation of the surfaces to be silvered, the silvering process, baking out, and the sealing off procedure are included. The principal features of the process are: (1) some modification of the concentration of the reagents in the Brashear formula for silvering, (2) methods of preventing the silver coat from being spoiled when heating to a high temperature during evacuation, (3) baking out in two stages of decreasing temperature, (4) pumping off the gases evolved while sealing off before completing the seal.

The effect of high temperatures on the reflectivity of the vacuum side of a silvered glass surface was investigated. From measurements of the rate of evaporation of liquid air and liquid hydrogen in a sample flask, it was found that the residual gas between the walls was responsible for one-fourth the heat transferred across the vacuum space of the flask filled with liquid air. Calculations based on these measurements yield a value of about $10^{-4} \mathrm{~mm}$ of mercury for the pressure of the residual gas, exclusive of $\mathrm{CO}_{2}$, at room temperature, and 0.97 as the average value of the reflectivity for the vacuum side of the silvered surfaces for temperature radiation of $300^{\circ} \mathrm{K}$.

\section{CONTENTS}

I. Introduction

Page

II. Preparation of the surfaces to be silvered.

935

III. Silvering

IV. Evacuation.

V. Discussion of the method

1. Silvering.

2. The effect of high temperatures on silvered surfaces

3. Evacuation

VI. Reflectivity of the silvered surfaces and density of residual gases.-

VII. Summary _....

VIII. Acknowledgments .

\section{INTRODUCTION}

Vacuum jacketed vessels, or Dewar flasks, made of Pyrex glass are less subject to breakage than those made of soft glass, hence they are to be preferred for use as containers of liquefied gases and for the insulation of low-temperature apparatus. It has been difficult to obtain Pyrex flasks that have insulating properties equal to those of similar flasks made of soft glass. With the help of information collected from several sources and by modifications of the usual methods, a technique has been developed by means of which it is possible to produce Pyrex flasks of uniformly good insulating quality. The insulating properties of the silvered flasks prepared in the way described in this paper are such that even in the wide-mouthed cylin- 
drical flasks filled with liquid air near to the lip, there is no boiling, the change of the liquid air to the gaseous phase taking place entirely by surface evaporation.

A description of the process finally used will be presented first, followed by a discussion of the method and the results of some tests.

\section{PREPARATION OF THE SURFACES TO BE SILVERED}

The space between the walls of the Dewar blank is filled with a potassium dichromate-sulphuric acid cleaning solution, and is maintained at a temperature of $60^{\circ} \mathrm{C}$. for about 30 minutes. After removing the cleaning solution and rinsing with tap water, concentrated nitric acid is shaken in the flask for about 10 minutes. Finally, the flask is washed, first with tap water and then two or three times with distilled water. It is best to prevent the walls from getting dry by allowing distilled water to remain in the flask until ready to silver.

If the temperature of a silvered flask is raised rapidly preparatory to its evacuation at high temperatures, the silvering is usually spoiled. The silver becomes spotted and sometimes scales off the surface. Although it is preferable to raise the temperature slowly, the spoiling of the silvering with rapid heating can, to a large extent, be overcome by etching the glass before silvering. The inside surfaces to be silvered are etched slightly with a 0.7 molal solution of hydrofluoric acid before treating the surfaces with the concentrated nitric acid after the cleaning solution has been rinsed out. The hydrofluoric acid is allowed to remain in the flask for 10 minutes, during which time the flask is shaken occasionally. The hydrofluoric acid is rinsed out with tap water and a dilute solution of ammonia is shaken in the flask to neutralize any remaining traces of the acid.

\section{SILVERING ${ }^{1}$}

Although the Brashear process is used for silvering, the concentrations of the solutions and the proportions in which they are mixed have been modified.

Three solutions are prepared in the following proportions:

A. $2 \mathrm{~L}$ of distilled water $+50 \mathrm{~g} \mathrm{Ag} \mathrm{NO}_{3}$.

B. $2 \mathrm{~L}$ of distilled water $+90 \mathrm{~g} \mathrm{KOH}$.

C. $(800 \mathrm{ml}$ of distilled water $+80 \mathrm{~g}$ cane sugar $)+(100 \mathrm{ml}$ of alcohol +3.5 $\mathrm{ml} \mathrm{HNO}_{3}$ specific gravity 1.42).

A should be kept in a dark place.

C improves with age.

The solutions are used in the proportions $A: B: C=16: 8: 1$ by volume.

Concentrated ammonium hydroxide is added to solution $\mathrm{A}$, drop by drop, while stirring, until the precipitate which first forms just completely disappears. To this is added the requisite amount of solution $B$, which causes a dark-brown or black precipitate to be formed. (If insufficient ammonium hydroxide was added before solution B was added, the precipitate will be yellowish green. If this occurs, the solution should be discarded and the process recommenced.) Ammonium hydroxide is added again, drop by drop, while stirring, until the

1 For general information on silvering, the reader is referred to Bureau of Standards Circular No. 389, The Making of Mirrors by the Deposition of Metal on Gless, which can be obtained from the Superintend- 
black precipitate is almost, but not completely, dissolved. If the solution is allowed to stand for about 30 minutes it will clear up except for some black flakes which settle to the bottom. The solution may be used immediately after the last addition of ammonium hydroxide, although it can stand for as long as an hour without affecting the results. ${ }^{2}$

The solution is decanted into the flask through the evacuating tube, filling the space between the walls about two-thirds full. The required amount of $\mathrm{C}$ is added and the solutions are mixed by shaking. The flask should be shaken or rolled while the silver is being deposited.

The temperature of the solution is kept as near $15^{\circ} \mathrm{C}$. as is practicable. The time required for the solution to react and deposit the silver on the glass walls depends, among other things, upon the age of the solution A; older solutions deposit silver more slowly. When $A$ is more than one month old the rate of deposition may be very slow, but it can be increased by adding in the final precipitation and redissolving of the combined $\mathrm{A}$ and $\mathrm{B}$ solutions with ammonium hydroxide, a little less ammonium hydroxide than would ordinarily be added, or by adding a small amount of solution A after the end point has been reached. The time of completion of the silvering process is ascertained by occasionally pouring out a little of the solution and noticing the character of the precipitate. When the precipitate becomes flocculent, the silvering is completed, and the solution is removed quickly in order to prevent the formation of the gray deposit, or bloom, which appears in spent solutions. The time of silvering varies from 10 to 30 minutes. A second coat of silver may be deposited over the first after removing the spent solution and rinsing. It is advantageous to silver a flask a second time in this manner if an opaque coat is not obtained the first time. Brighter and thicker silver coats can be obtained with a given amount of the solutions by silvering twice with half the amounts (diluting with water if desired) than can be obtained by silvering once with the full amount. After the silvering is completed the solution is washed out and as much as possible of the sediment adhering to the walls is removed by a vigorous shaking of the flask about half full of water. After a final rinsing with distilled water, the flask is dried by alternately exhausting and filling with dry air.

\section{EVACUATING}

The process used in evacuating and baking out the flasks was suggested by the results of some experiments of Langmuir ${ }^{3}$ on evacuating and baking out incandescent lamps.

In order to out-gas a flask, silvered or unsilvered, it is heated to a temperature near the softening range. Unless precautions are taken some portion of the flask may reach the softening range and the flask be ruined. The chance that such overheating may take place increases with the size of the flask. It was found that it is advantageous to roll the flask in a sheet of copper on the outside of which is wrapped a sheet of asbestos paper about one-sixteenth inch thick. The flask

2 There is danger of the formation of explosive compounds in ammoniacai silvering solutions after the

addition of the reducing solution. This is discussed in Bureau of Standards Circular No. 289.
Saul Dushman, Production and Measurement of High Vacuum, pp. 156-158. Publisher, General Electric Review, Schenectady, N. Y. 
is then placed horizontally on some pieces of insulating brick in an electric furnace and connected to a high vacuum system equipped with a McLeod gauge capable of detecting a pressure of $10^{-5} \mathrm{~mm}$ of mercury. Any water remaining in the flask is removed by evacuation with the fore pump alone. When this is completed, the mercury diffusion pump is started and a trap between the flask and the rest of the vacuum system is cooled with liquid air to prevent the diffusion of mercury vapor into the flask and its amalgamation with the silver.

When the pressure has been reduced to a value too small to be read on the McLeod gauge, the furnace is heated to $550^{\circ} \mathrm{C}$. Although the temperature of an unsilvered flask can be raised rapidly, the temperature of a silvered flask should be raised slowly, seven hours, at least, being allowed in reaching $550^{\circ} \mathrm{C}$. It was found convenient in the laboratory to raise the temperature to about $400^{\circ} \mathrm{C}$. on the day the out-gassing is started and to allow the flask to remain at this temperature over night, increasing it to $550^{\circ} \mathrm{C}$. the next morning. If the temperature of an unetched silvered flask is raised too quickly the silver is usually spoiled and in many cases some of the silver comes off the glass. For unsilvered flasks and silvered ones that are etched before silvering, the whole baking out and sealing off process can be completed in seven hours. The temperature of the flask is determined with two thermocouples, one of which is placed inside the flask and the other between the flask and the copper sheet. The current through the furnace is adjusted until the temperature inside and outside the flask is $550^{\circ} \mathrm{C}$., which should not be exceeded by more than a few degrees. Pumping is continued at this temperature for about an hour or until the pressure as read on the McLeod gauge shows no further decrease. The temperature is then allowed to fall to $400^{\circ} \mathrm{C}$. and the pumping continued at this temperature for at least 30 minutes. Then the flask while still hot is sealed off.

Care must be taken in sealing off the flask to allow the gases evolved during the sealing off process to be pumped out before the seal is completed. The following description of the sealing off procedure is copied here from page 81 of the Production and Measurement of High Vacuum, by Dushman.

Constrictions in glass vessels at points where they are to be sealed off after exhaust should not be too thick walled, otherwise a large body of glass will have to be heated during the seal off, causing the liberation of a great deal of gas. Furthermore, the constriction should be torched till it is almost melting and the pump allowed to exhaust the gas thus liberated for about two minutes, after which the sealing off should be performed as rapidly as possible without heating the glass any more than absolutely necessary.

The time required for removing the gases liberated before completing the seal will depend upon the size of the vacuum system and the speed of the pump. Four minutes were allowed for pumping out with the vacuum system used for this work.

After sealing off, the flask is removed from the furnace and cooled quickly in order to prevent further evolution of gases from the hot walls. This cooling may be effected by blowing air over the flask by means of an electric fan. 


\section{DISCUSSION OF THE METHOD}

\section{SILVERING}

The silvering formula is the result of adjusting the proportions and concentrations of the reagents in the standard Brashear process. The conditions under which Dewar flasks are silvered are different from those generally met with in silvering mirrors. A difficulty which presents itself in the silvering of Dewar flasks is the removal through the small evacuating tube of the spent solution after the silvering is completed. This may require several minutes, during which time the silver surface tarnishes and a gray deposit, or bloom, collects on it. This bloom and tarnish form rapidly in solutions that deposit their silver quickly. The formula presented here is slow acting, so that the solution can be removed before there is any visible tarnishing and before an appreciable amount of bloom collects on the surface. It was also found that the appearance of the flasks silvered with the slow acting solutions recommended are in general better than those silvered with more rapidly acting solutions.

The recommended concentration of potassium hydroxide is the average of the lowest and highest concentrations. which were found by trial to be just usable under the prevailing conditions. It was also found that adding sufficient ammonium hydroxide to completely clear up the first precipitate before the potassium hydroxide solution is added yielded more uniformly favorable results than additions of insufficient amounts.

Bureau of Standards Circular No. 389 recommends the addition of the potassium hydroxide directly to the silver nitrate solution, before adding any ammonium hydroxide. The same procedure may be used with the formula given in this paper, following the directions given in Circular No. 389 for clearing up the precipitate.

\section{THE EFFECT OF HIGH TEMPERATURES ON SILVERED SURFACES}

It frequently happens that heating a silvered surface in vacuo to a high temperature spoils its appearance. The silver becomes gray and appears to be thinner in parts and not infrequently scales off the glass. This, it has been found, is not due to any specific effect of the high temperature but is brought about by raising the temperature too rapidly. It can, to a large extent, be prevented by etching before silvering, but this is not necessary if the temperature is raised slowly enough. Before heating to a high temperature, the glass side of the silver coat of an etched Dewar flask is duller than that of an unetched flask, and although its appearance improves during the baking out, the outside appearance of the finished flask is not as good as that of the unetched flask. No difference was observed between the insulating properties of etched and unetched flasks.

In order to determine whether the high temperatures used in baking out flasks impair the reflectivity of the vacuum side of the silvered surface, which is the side effective in reducing heat transfer, eight glass plates, cut from a single larger plate of pyrex glass, were silvered and their reflectivities for visible light measured before and after heating in vacuo to high temperatures. The plates were silvered in a tray following as closely as possible the procedure used in silvering unetched flasks. The reflectivities for diffuse illumination of the 
air side of the silver surfaces, which were simply dried and not rubbed or polished, were measured normal to the surfaces with a Martens photometer using a blue, a green, and a red filter.

The plates were placed in a glass tube and baked out at high temperatures: 4 at $500^{\circ} \mathrm{C}$. for 3 hours, and the other 4 at $400^{\circ} \mathrm{C}$. for 40 hours, after which these same 4 were baked out at $550^{\circ} \mathrm{C}$. for 3 hours. The average values of the reflectivities of these 2 sets of 4 plates are given in Table 1.

TABLE 1

\begin{tabular}{|c|c|c|c|c|c|}
\hline \multirow{3}{*}{ Filter } & \multicolumn{5}{|c|}{ Reflectivity } \\
\hline & \multicolumn{3}{|c|}{ Set I, 4 plates } & \multicolumn{2}{|c|}{ Set II, 4 plates } \\
\hline & $\begin{array}{l}\text { Before } \\
\text { baking }\end{array}$ & $\begin{array}{c}\text { After } \\
\text { baking } \\
\text { for } 40 \\
\text { hours at } \\
400^{\circ} \mathrm{C} \text {. }\end{array}$ & $\begin{array}{c}\text { After an } \\
\text { additional } \\
3 \text { hours at } \\
550^{\circ} \mathrm{C} .\end{array}$ & $\begin{array}{l}\text { Before } \\
\text { baking }\end{array}$ & 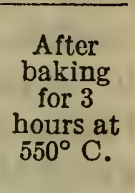 \\
\hline 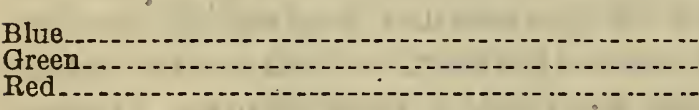 & $\begin{array}{r}0.34 \\
.56 \\
.75\end{array}$ & $\begin{array}{r}0.42 \\
.69 \\
.85\end{array}$ & $\begin{array}{r}0.57 \\
.79 \\
.92\end{array}$ & $\begin{array}{r}0.36 \\
.59 \\
.77\end{array}$ & $\begin{array}{r}0.59 \\
.82 \\
.94\end{array}$ \\
\hline
\end{tabular}

These observations show that heating to a high temperature improves the reflectivity of the vacuum side of a silvered surface for visible light and that the higher the temperature, the higher the reflectivity becomes. Of course, it is the infra-red and not the visible spectrum that is important in determining the transfer of heat between the walls of a silvered fiask, the wave length of maximum energy density for black body radiation at $300^{\circ} \mathrm{K}$. being $9.6 \mu$, but the measurements do make it seem very probable that if the high temperatures affect the reflectivity for $10 \mu$ radiation the result is an improvement rather than otherwise.

\section{EVACUATION}

The reasons for evacuating and baking out at $400^{\circ} \mathrm{C}$. after a similar procedure at $550^{\circ} \mathrm{C}$. are discussed by Dushman. ${ }^{4}$ Briefly they are as follows: At temperatures near the softening range, glass undergoes marked changes and large quantities of gas diffuse from the interior out through the surface. While the gases are thus diffusing their concentration at the surface is large. If the flask is cooled rapidly from these high temperatures and sealed off, much of the gas at the surface is retained by the glass for a time, only to be given up slowly after sealing off. Although there is a small rate of diffusion from the interior of pyrex glass at $400^{\circ} \mathrm{C}$., nevertheless gases at the surface can be effectively removed by baking for a short time at this temperature. It is not sufficient to remove only the gases adsorbed on the surface because, after sealing off, gases from the interior will diffuse slowly into the evacuated space.

Although the rate at which gases diffuse from the interior of glass at $400^{\circ} \mathrm{C}$. is small as compared with the rate at $550^{\circ} \mathrm{C}$., it is nevertheless appreciable, so that it is possible to produce good Dewar flasks by baking them out at $400^{\circ} \mathrm{C}$. for long periods of time. In

\footnotetext{
- Saul Dushman-Reference cited.
} 
a paper by Phipps, Copley, and Shaw ${ }^{5}$ which appeared while this paper was being prepared for publication, baking out at $400^{\circ} \mathrm{C}$. for 36 hours was recommended. It was decided to withhold publication until a comparison of the two methods could be made. This was done by preparing a number of silvered flasks, some by baking out at $550^{\circ}$ and $400^{\circ} \mathrm{C}$. as described above and the others at $400^{\circ} \mathrm{C}$. for 40 hours. In order to eliminate differences due to a possible nonuniformity in the silver deposits, some of those baked out at $400^{\circ}$ C. were afterwards baked out at $550^{\circ}$ and $400^{\circ} \mathrm{C}$. as recommended in this paper. In every case the flasks prepared by baking out at $550^{\circ}$ C. and afterwards at $400^{\circ} \mathrm{C}$. were slightly better. It was not determined whether this was because of a better vacuum or a possible improvement of the reflecting power of the silver at the higher temperature.

Baking out at the higher temperature has the further advantage that it requires less time which is especially favorable in the case of unsilvered and silvered flasks that have been etched. It has usually been considered that in baking out at temperatures above $400^{\circ} \mathrm{C}$. there was danger in that the flask might collapse and that its silver coat would be impaired, but these troubles can be easily overcome by surrounding the flask with copper and asbestos, and by raising its temperature slowly or by etching.

\section{REFLECTIVITY OF THE SILVERED SURFACES AND DENSITY OF RESIDUAL GASES}

In order to obtain approximate values of the reflectivity of the vacuum side of the silver deposit for temperature radiation, and the heat transferred by the residual gas between the walls, measurements were made of the rate of evaporation of liquid hydrogen and liquid air in a flask of about 1-liter capacity. These measurements made it possible to obtain separate values for the heat transferred by radiation and the heat transferred by the residual gas, since with liquid hydrogen in the flask the pressure of the residual gas is negligible and all the heat which crosses the vacuum space may be assumed to be transferred by radiation.

In order to reduce the flow of heat down from the lip, a flask with a long slender neck (15 cm long, $2.5 \mathrm{~cm} \mathrm{I.} \mathrm{D.)} \mathrm{was} \mathrm{chosen.} \mathrm{The}$ flask was silvered and evacuated according to the process described above. The rate of evaporation was determined by measuring with a gas meter the volume of gas which evaporated during measured intervals of time. When the temperature of the outer wall was $29.5^{\circ}$ C. liquid hydrogen evaporated at the rate of $3.0 \mathrm{~g}$ per hour and liquid air at the rate of $9.3 \mathrm{~g}$ per hour (period). For spherical flasks with long necks in which the residual gas pressure is small, of the order of $10^{-4} \mathrm{~mm}$, the rate of evaporation was found to be independent of the depth of the liquid in the spherical bulb. This suggests that the entire inner bulb was approximately at the temperature of the liquid and that all the heat transferred across the vacuum space to the spherical bulb was absorbed by the liquid and subsequent computations were based on this assumption. The evaporated gases take up in the neck the major part of the heat which flows down from the lip and the heat which is transferred across the vacuum space

6 T. E. Phipps, M. J. Copley, and E. J. Shaw, J. A. C. S., vol. 53, No. 4, p. 1365. 
surrounding the neck. From a measurement of the temperature gradient along the inside neck of the flask an approximate value was obtained for the heat which flowed by conduction from the neck into the spherical bulb. It amounted to less than 5 per cent of the heat transferred to the spherical bulb by radiation and was considered small enought to be neglected in this approximate calculation.

When the flask was filled with liquid hydrogen the gas pressure between the walls was so small that the heat transferred by gas between the walls could be neglected. (At liquid hydrogen temperatures, it is estimated that the vapor pressure of nitrogen is of the order of $10^{-10}$ $\mathrm{mm}$ of $\mathrm{Hg}$ and that of oxygen is even lower.) Using the value 108 calories per gram as the latent heat of vaporization of liquid hydrogen the energy reaching the inner bulb of the flask was found to be $7.7 \times 10^{3}$ ergs per sec for each $\mathrm{cm}^{2}$ of surface. Assuming that all this heat is transferred by radiation, the emissivity of the silvered surfaces may be calculated by the equation:

$$
\frac{e \sigma}{2-e}\left(T_{2}^{4}-T_{1}^{4}\right)=A
$$

where $e=$ emissivity of the surface which is assumed to be independent of the temperature,

$\sigma=$ Stefan-Boltzmann radiation constant $=5.72 \times 10^{-5} \mathrm{erg} \mathrm{cm}^{-2}$ degree $^{-4} \sec ^{-1}$,

$T_{1}=$ Absolute temperature of the inner surface $=20^{\circ} \mathrm{K}$.,

$T_{2}=$ Temperature of the outer surface $=302.5^{\circ} \mathrm{K}$.,

$A=$ Energy absorbed per $\mathrm{cm}^{2}$ of inner bulb, per sec $=7.7 \times 10^{3}$ ergs.

Substituting the values for the constants in the equation and solving for $e$, a value of 0.03 is obtained which corresponds to a reflectivity of 0.97 for temperature radiation of $300^{\circ} \mathrm{K}$. The value found in the International Critical Tables for the reflectivity of a polished freshly prepared film for $10 \mu$ radiation is 0.99 , which is the highest value found in these tables for the reflectivity of silver. Both to shorter and longer wave lengths the coefficient decreases a little.

Using the value 48.5 calories per gram as the latent heat of vaporization of liquid air, the heat transferred across the vacuum space when the flask contained liquid air was found to be 1.4 times as great as when the flask contained liquid hydrogen, the temperature of the outside surfaces of the flask being the same for both liquid air and liquid hydrogen. It is found therefore that about 29 per cent of the energy responsible for the evaporation of the liquid air was transferred by the residual gases.

Knowing the energy transferred by the residual gases when the flask.was filled with liquid air, a calculation, based upon Knudsen's theory of the transfer of heat by rarified gases, ${ }^{6}$ was made of the density of the residual gas, which was assumed to be principally nitrogen. Assuming an accommodation coefficient of unity, the density of the residual gas is found to be $1.2 \times 10^{-10} \mathrm{~g}$ per $\mathrm{cm}^{3}$ which corresponds to a pressure of $8 \times 10^{-5} \mathrm{~mm}$ of $\mathrm{Hg}$ at room temperature. Assuming an accommodation coefficient of 0.9 , which is more nearly the actual value, the density is $1.5 \times 10^{-10} \mathrm{~g}$ per $\mathrm{cm}^{3}$ and the corresponding

- L. B. Loeb, Kinetic Theory of Gases, Ed. 1, pp. 201-218. Publisher, McGraw Hill Book Co. 
pressure at room temperature is $1.0 \times 10^{-4} \mathrm{~mm}$ of $\mathrm{Hg}$. The vapor pressure of carbon dioxide at liquid air temperature is about $6 \times 10^{-6}$ $\mathrm{mm}$ of $\mathrm{Hg}$. Consequently the percentage concentration of $\mathrm{CO}_{2}$ at liquid air temperature is small but it is not possible to tell from these measurements what the concentration of $\mathrm{CO}_{2}$ is at room temperature.

From these simple measurements and computations, one can see that it is not physically impossible to make better flasks than the one here described. If the reflectivity could be increased to 99 per cent, the highest value given in the International Critical Tables for any silver surface, and the vacuum could be made perfect, the rate of evaporation of liquid air would be reduced to about one-fourth of the value given above. In the case of liquid hydrogen the rate of evaporation would be about one-third of the value given.

\section{SUMMARY}

A method has been described for silvering and evacuating Pyrex Dewar flasks by which it is possible to produce flasks with very good insulating properties equal to those of soft glass flasks.

\section{ACKNOWLEDGMENTS}

The authors take this opportunity to acknowledge the helpful suggestions made by Dr. M. F. Peters and F. A. Case.

Washington, July 29, 1931. 